\title{
Mechanical and thermal properties of PLA/halloysite bio-nanocomposite films: effect of halloysite nanoclay concentration and addition of glycerol
}

\begin{abstract}
The usage of biopolymers in developing biodegradable materials for applications that meet demands in society for sustainability and environmental safety has been limited due to the poor mechanical and thermal properties of biopolymers. This study aimed to improve the limited properties of biopolymers, particularly polylactic acid (PLA) films, by investigating the effect of incorporating different concentrations (0-5 wt.\%) of halloysite nanoclay and by adding glycerol plasticiser on the mechanical properties (tensile strength, elongation at break, Young's modulus, and toughness) and thermal properties (glass temperature (Tg), melting temperature $(\mathrm{Tm})$, and crystalline temperature $(\mathrm{Tc})$ ) of the produced bio-nanocomposite films. It was found that the addition of halloysite nanoclay and glycerol improved the mechanical and thermal properties of the films. PLA films incorporated with $3 \mathrm{wt} \%$ concentration of halloysite nanoclay resulted in optimum mechanical properties due to the uniform distribution or dispersion of halloysite nanoclay. The addition of halloysite nanoclay and glycerol reduced the $\mathrm{Tg}, \mathrm{Tm}$, and $\mathrm{Tc}$ of the films, suggesting that they can improve the processability of the biopolymer. The bio-nanocomposite films produced in this work have the potential to replace non-biodegradable films due to the improved properties of the films.
\end{abstract}

Keyword: Bio-nanocomposite; Halloysite; Mechanical properties; Polylactic acid; Thermal properties 